\title{
Dynamic Infrared Thermography (DIRT) in Biomedical Applications: DIEP Flap Breast Reconstruction and Skin Cancer ${ }^{\dagger}$
}

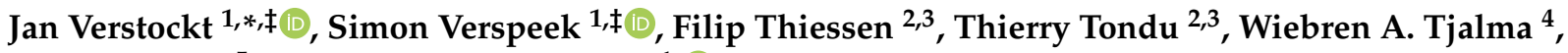 \\ Lieve Brochez ${ }^{5}$ and Gunther Steenackers ${ }^{1}$ (D)
}

check for updates

Citation: Verstockt, J.; Verspeek, S.; Thiessen, F.; Tondu, T.; Tjalma, W.A.; Brochez, L.; Steenackers, G. Dynamic Infrared Thermography (DIRT) in Biomedical Applications: DIEP Flap Breast Reconstruction and Skin Cancer. Eng. Proc. 2021, 8, 3. https://doi.org/10.3390/engproc 2021008003

Academic Editors: Giovanni Ferrarini, Paolo Bison and Gianluca Cadelano

Published: 17 November 2021

Publisher's Note: MDPI stays neutral with regard to jurisdictional claims in published maps and institutional affiliations.

Copyright: (C) 2021 by the authors. Licensee MDPI, Basel, Switzerland. This article is an open access article distributed under the terms and conditions of the Creative Commons Attribution (CC BY) license (https:// creativecommons.org/licenses/by/ $4.0 /)$.
1 InViLab Research Group, University of Antwerp, Groenenborgerlaan 171, B-2020 Antwerp, Belgium; simon.verspeek@uantwerpen.be (S.V.); gunther.steenackers@uantwerpen.be (G.S.)

2 Department of Plastic, Reconstructive and Aesthetic Surgery, Multidisciplinary Breast Clinic, Antwerp University Hospital, University of Antwerp, Wilrijkstraat 10, B-2650 Antwerp, Belgium; filip.thiessen@clinic12b.be (F.T.); t.tondu@clinic12b.be (T.T.)

3 Department of Plastic, Reconstructive and Aesthetic Surgery, Ziekenhuis Netwerk Antwerpen, Lindendreef 1 , B-2020 Antwerp, Belgium

4 Gynaecological Oncology Unit, Department of Obstetrics and Gynaecology, Multidisciplinary Breast Clinic, Antwerp University Hospital, University of Antwerp, Wilrijkstraat 10, B-2650 Antwerp, Belgium; wiebren.tjalma@uza.be

5 Department of Dermatology, Ghent University Hospital, C. Heymanslaan 10, B-9000 Ghent, Belgium; lieve.brochez@uzgent.be

* Correspondence: jan.verstockt@uantwerpen.be

+ Presented at the 16th International Workshop on Advanced Infrared Technology \& Applications, 26-28 October 2021; Available online: https:/ / aita2021.sciforum.net/.

$\ddagger$ These authors contributed equally to this work.

Abstract: Infrared thermography technology has improved drastically in recent years and is regaining interest in medicine for applications such as deep inferior epigastric perforate flap breast reconstruction, breast cancer diagnosis, skin tissue identification, psoriasis detection, etc. However, there is still a need for an optimised measurement setup and protocol in order to capture the most suitable images for decision making and further processing. Nowadays, different cooling methods are being used; nevertheless, a general optimised cooling protocol is not yet defined. In this manuscript, several cooling techniques, as well as the measurement setups, are reviewed and optimised. It is possible to enhance the thermal images by selecting an appropriate cooling method and duration, and additionally, an optimised measurement setup enables a comparison between different inspections.

Keywords: dynamic infrared thermography; optimisation; skin tissue identification; deep inferior epigastric perforator flap breast reconstruction

\section{Introduction}

Infrared thermography (IRT) has been continuously improved over the last few years and has found widespread applications within scientific research and industry environments [1]. IRT in medicine has gained interest for use in deep inferior epigastric perforate (DIEP) flap breast reconstruction [2], psoriasis [3], skin cancer [4], breast cancer and many other medical conditions [5].

In our biomedical IRT research, we focus on DIEP flap breast reconstruction and skin tissue identification for skin cancer diagnosis. In breast reconstructions with DIEP flaps, the skin and subcutaneous tissue from the patient's lower abdomen are used as a free flap to reconstruct the breast. One of the key elements in DIEP flap surgery is the selection of the best perforator to obtain sufficient blood supply to the flap after anastomosis [6]. The diagnosis of skin lesions measures the thermal response to thermal stress (cold provocation). 
After cooling the skin surface, a different thermal behaviour can be displayed because of the difference in the thermophysical properties of the lesion and the healthy tissue [7].

\subsection{Thermal Provocation for Dynamic Infrared Thermography}

\subsubsection{DIEP Flap Breast Reconstruction}

During the peroperative stage, to locate the best perforators, a cold challenge is performed on the abdomen. The first occurring hot spots during rewarming can be related to perforators with a larger blood supply and thus can be used for anastomosis of the DIEP flap [8]. The introduction of a cold challenge is necessary to create an equal starting situation for the whole area of interest. Different cooling methods have already been tested: Weum et al. [9] used a desktop fan. Thiessen et al. [6] did a comparison between different cooling methods. Cooling with a desktop fan did not meet their requirements of even cooling the surface and did not extract enough heat from the abdomen.

During the peroperative measurements, the influence of a specific perforator will determine which areas of the abdominal flap will be perfused by which dominant perforator and influences the final choice of perforators used for reconstruction. This is demonstrated in Figure 1. After anastomosis, the blood flow in the DIEP flap can be checked with DIRT to verify if the flap is properly vascularised to reduce the chance of necrosis.

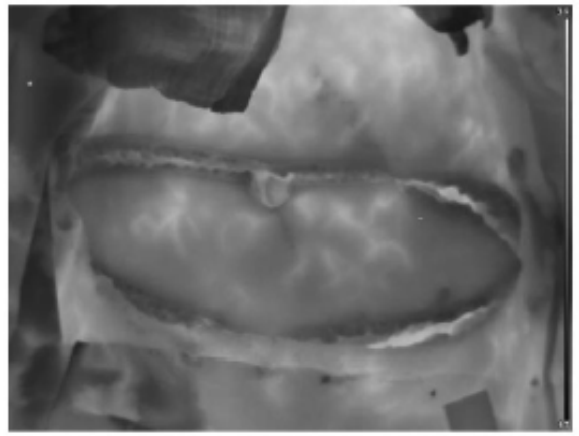

(a)

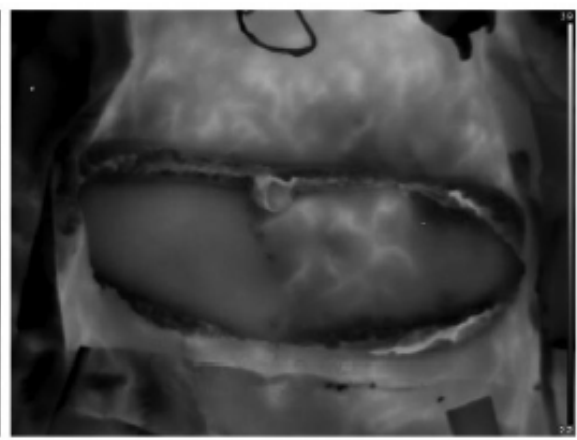

(b)

Figure 1. Peroperative measurement. (a) Perforators A and B 4 min after the clamp was removed. (b) Only perforator B 4 min after the clamp was removed. The influence of perforator A on the flap can be deduced by subtracting the influence of perforator B in subfigure (a).

Postoperative measurements include a static image of the reconstructed breast and a dynamic recording after the introduction of a cold challenge. These measurements can help visualize arterial or venous thrombosis and potential necrotic area(s) before clinical observation.

\subsubsection{Skin Tissue Identification}

The cooling of the skin lesion is necessary to create a thermal contrast to be able to differentiate between unhealthy and healthy skin. Magelhaes et al. [10] cool the lesions with the use of an aluminium medal. Godoy et al. [11] tested a Ranque-Hilsch vortex tube but replaced it with a commercially available AC unit due to its portability and ease of use.

\subsection{Measurement Setup}

\subsubsection{DIEP Flap Breast Reconstruction}

Weum et al. [9] describe in their work the use of an IR camera mounted on a tripod with an overhanging beam to do preoperative measurements. In their work, no measurement setup is described for peroperative measurements.

\subsubsection{Skin Tissue Identification}

During measurements for skin cancer identification, the IR camera is mostly mounted on a tripod [4]. Involuntary body movements are difficult to suppress while having a 
complex body position or while standing straight. This leads to degrading imaging quality. Magelhaes et al. [10] make use of a hand-held IR device that solves the problem of hardto-reach skin lesions but makes it difficult to keep the measuring parameters, such as the distance to the object or angle, constant.

This manuscript focuses on the steps taken towards an optimised thermography measurement setup for biomedical applications. On one hand, the external stimulation of the body has a remarkable influence on the induced thermal contrast and should therefore be optimised. On the other hand, the used measurement hardware affects the possibility of performing accurate and consistent measurements. A standardised inspection protocol and setup enables comparing data sets between different measurements.

\section{Materials and Methods}

\subsection{DIEP Flap Breast Reconstruction}

During the DIEP flap breast reconstruction, the measurements are performed using a long-wave thermal camera mounted on a long beam tripod, as can be read in [2]. The used camera is the Xenics Gobi 640 camera, which is a microbolometer working in the 8-14 $\mu \mathrm{m}$ range. The camera is mounted perpendicular to the patient's abdomen in order to obtain a clear view of the whole region of interest. The abdomen is cooled with a bag of ice water for $3 \mathrm{~min}$, and afterwards, images are captured at $50 \mathrm{~Hz}$ for $5 \mathrm{~min}$. The plastic bag cools down the abdomen correctly, without creases, if handled with care. Test measurements to cool the abdomen with a balloon filled with ice water are in progress. This technique has the advantage that no creases can form during cooling in comparison to a sterile plastic bag, as shown in Figure 2.



a)

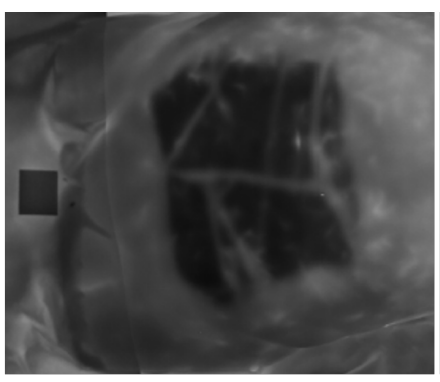



Figure 2. (a) Cooling of the abdomen with a balloon filled with water and ice. (b) Creases and uneven temperature after cooling with a sterile plastic bag filled with water and ice. (c) No creases and even temperature after cooling with a balloon filled with water and ice. Light coloured line is a scar from previous surgery.

\subsection{Skin Tissue Identification}

In the context of skin tissue identification measurements, several thermal cameras have been compared: the Xenics Gobi 640 camera, as used in Section 2.1, as well as a FLIR A700 camera. Both cameras function in the long-wave infrared spectrum between 8 and $14 \mu \mathrm{m}$. The camera is mounted on a stand specifically designed and built for biomedical applications, as can be seen in Figure 3. The vertical linear guide rails at the end of the mount arm makes it possible to keep the camera located at a fixed distance from the skin. This is especially necessary if the FLIR A700 is used in combination with a macro lens. The region of interest containing the abnormal skin lesion is indicated using a semi-flexible leather square or plastic marker to make the ROI visible in the IR region. Different skin cooling methods are used, such as fan cooling, cooling with an aluminium plate, and cooling with a gel pack for various cooling times between $30 \mathrm{~s}$ and $90 \mathrm{~s}$. 


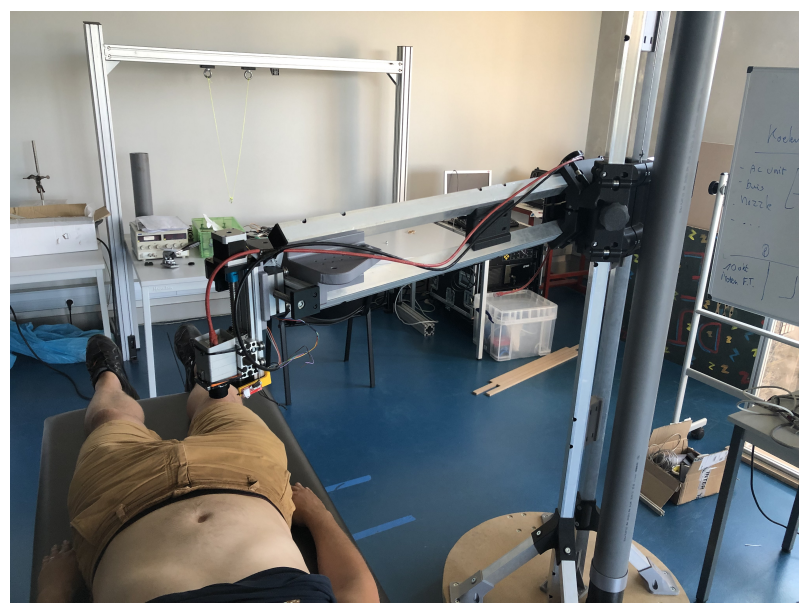

Figure 3. Image taken during skin tissue identification measurements. The thermal camera is mounted on the linear guide rails at the end of the mount arm. The mount enables the camera to be moved to any wanted location above the patients body, and the linear guide rails provide the possibility of keeping a fixed distance between the camera lens and the skin.

\section{Conclusions}

In order to be able to compare measurements taken at different moments, a generalised optimal measurement setup and protocol is necessary. Comparing different cooling techniques and cooling times resulted in finding the best suitable cooling protocol for thermal measurements during DIEP flap breast reconstruction surgery and for skin tissue identification. For both medical procedures, the measurement hardware was reviewed and optimised in order to increase the accuracy and repeatability of the measurements. For DIEP flap breast reconstructions, the hardware setup consists of only a long beam tripod resulting in static measurements, but for skin tissue identification, the mount is accommodated with a linear guide in order to provide a fixed measurement distance automatically.

Funding: This research was funded by the FWO via support for the FWO research project, "Optimized skin tissue identification by combined thermal and hyperspectral imaging methodology." (project number 41882 (FWO G0A9720N)). The researchers received funding from the Antwerp University IOF-council through project PSID-34924 entitled "Fast Broadband Lock-In Thermography for Fragile Structures Using System Identification."

Institutional Review Board Statement: The study was approved by the local ethics committee (B300201941125) and was carried out according to the Declaration of Helsinki (1983).

Informed Consent Statement: Informed consent was obtained from all subjects involved in the study.

Data Availability Statement: No new data were created or analyzed in this study. Data sharing is not applicable to this article.

Conflicts of Interest: The authors declare no conflict of interest.

\section{References}

1. Ring, E.F.J.; Ammer, K. Infrared thermal imaging in medicine. Physiol. Meas. 2012, 33, R33-R46. [CrossRef] [PubMed]

2. Thiessen, F.E.F.; Vermeersch, N.; Tondu, T.; Van Thielen, J.; Vrints, I.; Berzenji, L.; Verhoeven, V.; Hubens, G.; Verstockt, J.; Steenackers, G.; et al. Dynamic Infrared Thermography (DIRT) in DIEP flap breast reconstruction: A clinical study with a standardized measurement setup. Eur. J. Obstet. Gynecol. Reprod. Biol. 2020, 252, 166-173. [CrossRef] [PubMed]

3. Strakowska, M.; Strąkowski, R.; Strzelecki, M.; De Mey, G.; Więcek, B. Thermal modelling and screening method for skin pathologies using active thermography. Biocybern. Biomed. Eng. 2018, 38, 602-610. [CrossRef]

4. Cheng, T.Y.; Herman, C. Analysis of skin cooling for quantitative dynamic infrared imaging of near-surface lesions. Int. J. Therm. Sci. 2014, 86, 175-188. [CrossRef]

5. Lahiri, B.B.; Bagavathiappan, S.; Jayakumar, T.; Philip, J. Medical applications of infrared thermography: A review. Infrared Phys. Technol. 2012, 55, 221-235. [CrossRef] [PubMed] 
6. Thiessen, F.E.F.; Tondu, T.; Vermeersch, N.; Cloostermans, B.; Lundahl, R.; Ribbens, B.; Berzenji, L.; Verhoeven, V.; Hubens, G.; Steenackers, G.; et al. Dynamic infrared thermography (DIRT) in Deep Inferior Epigastric Perforator (DIEP) flap breast reconstruction: standardization of the measurement set-up. Gland. Surg. 2019, 8, 799-805. [CrossRef] [PubMed]

7. Herman, C. The role of dynamic infrared imaging in melanoma diagnosis. Expert Rev. Dermatol. 2013, 8, 177-184. [CrossRef] [PubMed]

8. Verstockt, J.; Thiessen, F.; Thiessen, F.; Cloostermans, B.; Tjalma, W.; Steenackers, G. DIEP flap breast reconstructions: thermographic assistance as a possibility for perforator mapping and improvement of DIEP flap quality. Appl. Opt. 2020, 59, E48-E56. [CrossRef] [PubMed]

9. Weum, S.; Mercer, J.B.; de Weerd, L. Evaluation of dynamic infrared thermography as an alternative to CT angiography for perforator mapping in breast reconstruction: a clinical study. BMC Med. Imaging 2016, 16, 43. [CrossRef] [PubMed]

10. Magalhaes, C.; Vardasca, R.; Rebelo, M.; Valenca-Filipe, R.; Ribeiro, M.; Mendes, J. Distinguishing melanocytic nevi from melanomas using static and dynamic infrared thermal imaging. J. Eur. Acad. Dermatol. Venereol. 2019, 33, 1700-1705. [CrossRef] [PubMed]

11. Godoy, S.E.; Hayat, M.M.; Ramirez, D.A.; Myers, S.A.; Padilla, R.S.; Krishna, S. Detection theory for accurate and non-invasive skin cancer diagnosis using dynamic thermal imaging. Biomed. Opt. Express 2017, 8, 2301. [CrossRef] [PubMed] 